\title{
A atenção à saúde do idoso: ações e perspectivas dos profissionais*
}

\section{Healthattertion to the ddaly: ations and perspectives of professionals}

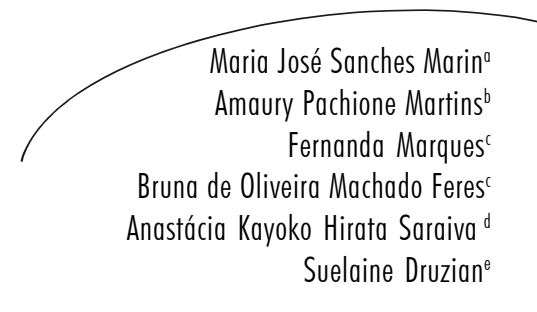

\section{Resumo}

O crescente aumento da população idosa e os complexos problemas de saúde que a envolvem tornam evidente a necessidade de atenção adequada à sua saúde, e espera-se que a Estratégia de Saúde da Família (ESF) possa trazer contribuições. $\mathrm{O}$ presente estudo propõe-se a analisar a percepção de profissionais que atuam na ESF quanto à saúde do idoso, as ações que vêm sendo desenvolvidas e as perspectivas dos profissionais visando à promoção, prevenção, cura e reabilitação das condições de saúde deles. Trata-se de um estudo qualitativo, realizado por meio de entrevistas com dentistas, enfermeiros e médicos que atuam na ESF. A análise dos resultados possibilitou a identificação de três categorias temáticas: 1 . Falta de adesão ao cuidado e de apoio dos familiares; 2 . Assistência centrada no aspecto curativo e no atendimento a demanda; 3 . Vislumbre de mudança no modelo de atenção. Os dados revelam consonância entre as respostas dos entrevistados com o momento histórico e social das transformações que perpassam o modelo de atenção, levando a acreditar na ESF como um caminho de consolidação do Sistema Único de Saúde, desde que haja investimentos visando a instrumentalizar as equipes para atuar frente à nova lógica.

\section{Abstract}

The growing number of the elderly population and their complex health problems show the necessity of an adequate health care to the elderly, and it is expected that Family Health Strategy will bring contributions to them. This study aims at

\author{
Palavras-chave: \\ prática profissional; \\ pessoal de saúde; \\ equipe de assistência \\ ao paciente; saúde \\ do idoso; serviços \\ de saúde para \\ idosos; estratégias; \\ sistema único de \\ saúde; pesquisa \\ qualitativa
}

Programa de Pós-Graduação em Ciências da Saúde, Universidade Federal de São Paulo. São Paulo, Brasil.

Correspondência / Correspondence

Maria José Sanches Marin

Av. Brigadeiro Eduardo Gomes,1886

17514-000 - Marília, SP, Brasil

E-mail: marnadia@terra.com.br

*Projeto de Iniciação Científica financiado pela Fapesp - Parecer no 06/60058-9. 
analyzing the perception of the professionals who work at the Family Health Strategy about the elderly's health, the actions performed and the perspectives to promote, prevent, cure and rehabilitate the elderly's health.It is a qualitative study carried out by means of interviews with dentists, nurses and medical doctors who work at the Family Health Strategy. The analysis of results made allowed the identification of three theme categories: 1 . non adhesion to care and lack of family support; 2 . health assistance centered on cure according to health care protocols; 3 . a glimpse at changing the health care models. Data shows a consonance between the interviewed people and the historical and social transformations in the health care model; therefore it is possible to consider that the Family Health Strategy is a way to consolidate the Unified Health System, since it receives the tools for the teams to act according to its new logics.
Key words: professional practice; health personnel; patient care team; health of the elderly; health services for the aged; strategies; single health system; qualitative research
INTRODUÇÃOO

Profundas mudanças vêm ocorrendo na estrutura etária da população, caracterizada pela acentuada longevidade e atribuída às melhores condições de vida das pessoas, em especial no que se refere ao acesso às novas tecnologias de atendimento à saúde. ${ }^{1} \mathrm{No}$ Brasil, a população idosa perfazia, em 2002, um total de 14,1 milhões de pessoas e, para 2025, projeta-se um total de 33,4 milhões. Entre 1950 e 2025, a população idosa terá crescido 16 vezes contra cinco vezes a população total. ${ }^{2}$ Destaca-se na população idosa o aumento do número de pessoas com 80 anos ou mais, sendo essa a faixa etária de maior crescimento, tanto nos países desenvolvidos como nos países em desenvolvimento. ${ }^{3,4}$

Juntamente com as modificações da estrutura etária da população, constatam-se mudanças epidemiológicas, caracterizadas por doenças e fatores de risco relacionados com o estilo de vida. Sendo problemas de longa duração, estes tornam os idosos os principais usuários dos serviços de saúde. Tais serviços nem sempre estão devidamente preparados para atender às necessidades dessa parcela $\mathrm{da}$ população de forma a garantir qualidade de vida por meio da promoção, prevenção, cura e reabilitação da saúde. Em vista desse contexto, verifica-se a necessidade de transformação no modelo assistencial e iniciativas inovadoras de informação, educação e comunicação. ${ }^{5}$

Em nossa realidade, o modelo de atenção à saúde predominante por muitas décadas tem-se caracterizado pela fragmentação do cuidado, centralização do poder no profissional médico e dificuldade de acesso da população com menor poder aquisitivo a essa atenção. Desta forma, não se tem conseguido atender adequadamente aos diversos e complexos problemas de saúde da população.

A partir da década de 1980, há o reconhecimento, pela Constituição Federal brasileira, da saúde como direito fundamental do ser humano e a explicitação dos princípios doSUS, cuja operacionalização requer 
a articulação entre as ações de promoção, prevenção, recuperação e reabilitação, nas dimensões coletiva e individual da população.

Pautada no modelo de Vigilância à Saúde, propõe-se, na década de 1990, a Estratégia de Saúde da Família (ESF) como uma forma de reorientação dos serviços de saúde por meio do fortalecimento de práticas voltadas para a integralidade da atenção e ações intersetoriais, incluindo ações sobre as exposições, vulnerabilidades e necessidades dos indivíduos, ${ }^{6}$ o que possibilita aos profissionais da equipe a compreensão ampliada do processo saúde/doença e a necessidade de intervenção para além das práticas curativas. ${ }^{7}$

Espera-se da equipe de saúde da família, ao atuar em uma área adscrita, o desenvolvimento de ações de saúde, dirigidas às famílias e ao seu ambiente e com ênfase nos aspectos preventivos, curativos e de reabilitação, articuladas com outros setores que contribuem para melhoria das condições de saúde. ${ }^{8}$ Além disso, por essa estratégia, elegem-se como pontos centrais o estabelecimento de vínculo e a criação de laços de compromisso e de responsabilidade entre os profissionais de saúde e a população. ${ }^{9}$

Apesar dos avanços no que se refere aos princípios norteadores do SUS è̀s estratégias propostas, ainda se enfrenta uma série de dificuldades, destacando-se a fragmentação do processo de trabalho e das relações entre os diferentes profissionais; falta de complementaridade entre rede básica e o sistema de referência; precária interação nas equipes e despreparo para lidar com a di- mensão subjetiva nas práticas de atenção; baixo investimento na qualificação dos trabalhadores e no fomento à co-gestão e, ain$\mathrm{da}$, desrespeito aos direitos dos usuários. ${ }^{10}$

Visando a caminhar na perspectiva de superação destas dificuldades e na direcionalidade dos princípios constitucionais, o Ministério da Saúde aprova em 2006 o "Pacto pela Saúde", com a finalidade de pactuar novos compromissos e responsabilidades em nível federal, estadual e municipal, com ênfase nas necessidades de saúde da população, articulando três componentes básicos: Pacto pela vida, Pacto em Defesa do SUS e Pacto de Gestão doSUS. Neste documento, ao tratar do Pacto pela Vida, a saúde do idoso aparece como uma das seis prioridades pactuadas. ${ }^{11}$

No mesmo ano, foi aprovada a Política Nacional de Saúde da Pessoa Idosa, para estimular a formulação de estratégias capazes de dar conta da heterogeneidade da população idosa e, por conseguinte, da diversidade de questões apresentadas. Frente às diretrizes apontadas nesta política, a ESF continua sendo o espaço estratégico para o desenvolvimento das ações de saúde voltadas para os idosos. ${ }^{12}$

Assim, Silvestre e Costa Neto ${ }^{13}$ acrescentam que o cuidado comunitário do idoso deve basear-se na família e na atenção básica da saúde, pela possibilidade de maior reconhecimento dos problemas de saúde, e desenvolvimento de vínculo com o idoso .

Em vista disso, os profissionais que atuam naESF precisam estar cientes da responsabilidade imposta, além de estarem preparados 
para lidar com essa realidade. No entanto, temse observado que pouco tem sido feito no sentido de melhorar as condições de vida e saúde desta parcela da população que, pelas características de vulnerabilidade, demanda atenção que visa a atender às suas especificidades.

Além disso, referindo-se à atenção à saúde do idoso na ESF, identificam-se na literatura poucos estudos que abordam o tema, pois se tratade uma estratégia recente que ainda vem tentando se organizar do ponto de vista estrutural e em relação ao preparo dos profissionais para atuarem segundo a lógica da vigilância da saúde.

Estudo que se propõe a identificar o perfil de idosos atendidos em USF, em Aparecida de Goiânia (GO), aponta para a necessidade de preparar os profissionais para atuar na promoção à saúde, na prevenção de agravos, no tratamento e na reabilitação de doenças, em especial as crônico-degenerativas, visando à melhoria da qualidade de vida no domicílio, com autonomia e independência. ${ }^{14}$

Em outro estudo que busca analisar as concepções e práticas dos agentes comunitários de saúde na atenção à saúde do idoso, destaca-se a citação de dificuldades dos agentes na resolutividade das queixas e uma prática preponderantemente voltada para a marcação de exames e consultas, reforço da consulta médica e de enfermagem, além de controle da medicação e do tratamento. ${ }^{15}$

Ao estudar os fatores de risco e os problemas dos idosos atendidos em um PSF, os autores encontraram alta proporção de analfa- betismo $(43,1 \%)$; sedentarismo (60\%), vida solitária (22,4\%), além de alto índice de doenças cardiovasculares $(60,3 \%)$, presença de dor $(43,1 \%)$, sendo que $75 \%$ deles apresentavam três ou mais problemas de saúde. A intensidade de tais problemas leva à constatação de que eles também fazem uso de grande quantidade de medicamentos. ${ }^{16}$

Diante das evidentes necessidades de saúde apresentadas pelos idosos, das políticas e estratégias propostas como formas de melhorar a qualidade de vida das pessoas e da importância do compromisso dos profissionais de saúde frente a tais propostas, propomos para o presente estudo analisar a percepção de profissionais que atuam na ESF quanto à saúde do idoso, as ações que vêm sendo desenvolvidas e as perspectivas visando à promoção, à prevenção, à cura e à reabilitação das condições de saúde dessa parcela da população.

\section{METODOLOGIA}

Trata-se de um estudo qualitativo que se propõe a analisar a percepção de profissionais que atuam na ESF quanto à saúde do idoso, às ações que vêm sendo desenvolvidas e às perspectivas dos profissionais, no sentido de melhorar as condições de vida dos atendidos pelo programa, a partir da narrativa de sujeitos que compõem as equipes $d a$ ESF de um município de médio porte do interior paulista, visto que, por meio das narrativas em que as pessoas contam experiências, crenças e expectativas, anunciam-se novas possibilidades, intenções e projetos. ${ }^{17}$ 
O município onde o estudo foi realizado conta hoje com 28 equipes da ESF, as quais atendem aproximadamente a $40 \% \mathrm{da}$ população e estão instaladas em locais de maior carência socioeconômica e, portanto, de maior dificuldade de acesso dessa população aos serviços de saúde.

De maneira geral, as unidades cumprem com os requisitos mínimos necessários à sua implantação, conforme preconiza o Ministério da Saúde, em relação à estrutura física, composição da equipe mínima e desenvolvimento dos programas nacionais básicos.

Os sujeitos do estudo são os médicos, enfermeiros e cirurgiões dentista que compõem as equipes da ESF, considerando que tais profissionais são aqueles que, de forma articulada, coordenam as ações da equipe.

Foram sorteadas oito unidades, sendo duas de cada região da cidade (norte, sul, leste, oeste) compreendendo um total de 24 sujeitos. No entanto, quatro deles não foram entrevistados devido à indisponibilidade, mesmo após o segundo agendamento de horário para a entrevista. A amostra, portanto, foi constituída de 20 sujeitos.

Após sorteio das unidades, foi realizado contato telefônico e marcado horário para as entrevistas, que contaram com as seguintes questões norteadoras: Qual a sua percepção a respeito da saúde do idoso da sua área de abrangência? Quais as ações que você desenvolve na unidade visando a promoção, prevenção, tratamento e reabilitação da saúde do idoso? Que ações você considera que deveriam ser realizadas pela unidade onde atua, visando à promoção, prevenção, tratamento e reabilitação da saúde do idoso. As entrevistas foram gravadas em fita cassete e posteriormente transcritas.

O estudo contou com autorização da Secretaria de Saúde do Município e aprovação do Comitê de Ética em Pesquisa com Seres Humanos da Faculdade de Medicina da cidade. Os profissionais foram esclarecidos da finalidade do estudo e, quando de acordo em participar, assinaram o Termo de Consentimento Livre e Esclarecido.

Os dados foram submetidos à técnica de análise de conteúdo, que Bardin conceitua como "um conjunto de técnicas de análise das comunicacões visando obter, porprocedimentos sistemáticos e objetivos de descrição do conteúdo das mensagens, indicadores que permitam inferência de conhecimentos relativos às condicōes de producão / recepção destas mensagens" ${ }^{18}$ Entre as técnicas de análise de conteúdo, optou-se pela modalidade temática por ser uma das formas mais adequada à investigação qualitativa. ${ }^{19}$

A técnica de análise de conteúdo, modalidade temática, é desenvolvida em três etapas, compreendendo a pré-análise que pode ser decomposta em leitura flutuante do conjunto das comunicações; constituição do corpus, ou seja, organização do material de forma a responder a algumas normas de validade como a exaustividade, representatividade, homogeneidade e pertinência; formulação de hipótese e objetivos em relação ao material qualitativo; definição das unidades de registro. $\mathrm{Na}$ segunda etapa, realiza-se a codificação dos dados 
brutos visando a alcançar o núcleo de compreensão do texto. Na seqüência, propõe-se o tratamento dos resultados obtidos e interpretação a partir de inferências previstas no seu quadro teórico ou abrindo-se outras pistas em torno de dimensões teóricas sugeridas naleitura do material.

\section{RESULTADOS}

Ao analisar os resultados, tomando como parâmetro a fala dos profissionais entrevistados, foi possível construir três categorias temáticas:

\section{Falta de adesão ao cuidado e de apoio dos familiares}

Alguns dos profissionais entrevistados apontaram para a falta de adesão dos idosos e de apoio dos familiares às ações de saúde, parecendo atribuir a ambos a responsabilidade pelo não controle da saúde, conforme descrito a seguir:

“...os idosos são bem assistidos na nossa unidade..., mas acho que eles mesmos estão deixando de desenvolver seu autocuidado, falta um despertar deles para que eles próprios cuidem da sua saúde. Nós estamos à disposição deles aqui, para orientar, tratar...."(A3)

"...eles não são controlados, os que têm pressão alta, diabetes não são controlados, eles têm muitas medicações, quase que para tudo, mas não aderem ao tratamento." (A2)

"Eupercebo é que são idosos com uma carência afetiva, com um descompromisso familiar em relação à saúde dos idosos, pela dificuldade que eu estou vendo pela adesão ao tratamento medicamentoso."(B2)

A adesão, no entanto, é um aspecto ligado às relações, muito mais do que a fatores individuais. Ao resgatar o conceito de adesão, pode-se constatar que, do ponto de vista etimológico, do latim adhaesione, o termo significa junção, união, aprovação, acordo, manifestação de solidariedade, apoio; pressupõe relação e vínculo. Entende-se ser um processo multifatorial, estruturado a partir da relação entre quem cuida e quem é cuidado e envolve constância, perseverança e freqüência. ${ }^{20}$

A adesão ao tratamento é um aspecto que vem merecendo considerável atenção nos últimos anos, uma vez que envolve significante custo social, pessoal e financeiro. Estima-se que 20 a $80 \%$ dos pacientes não aderem de fato ao regime terapêutico. A não adesão indica que os pacientes não são recipientes passivos e que o termo também indica que o tratamento deve ser oferecido num contexto de "aliança terapêutica". ${ }^{21}$ Outro estudo demonstra que o relacionamento terapêutico ineficaz está fortemente relacionado com a não-adesão. ${ }^{22} \mathrm{~A}$ adesão demanda a construção de uma consciência cidadã e, esta, por sua vez, "não é algo natural”, posto que está submetida a regras sociais e culturais. Nas sociedades coletivistas, como esta em que vivemos "o todo prevalece sobre as partes, a hierarquia é o princípio básico e o que vale é a relação". ${ }^{23}$

Os casos de resistência revelam, muitas vezes, que as pessoas estão se sentindo des- 
valorizadas ou não está havendo um verdadeiro diálogo entre os saberes da equipe e dos usuários. Destaca-se, ainda, que na confrontação de saberes éimportante considerar a complexidade articulada no núcleo de histórias e a cultura que as explicita, obtendo-se, assim, a explicação para a dificuldade de adesão. ${ }^{20}$

Conforme apontado nas falas dos sujeitos é importante considerar, ainda, os aspectos relacionados ao próprio idoso, incluindo crenças, valores e papel social. Nas últimas décadas, tem-se constatado que o idoso vem assumindo diferentes responsabilidades, tanto no provimento de recursos financeiros para a família, por meio da aposentadoria, como no cuidado com os netos, levando muitas vezes a dificuldades financeiras e ao estresse físico e emocional, que dificultam o investimento na promoção da saúde e prevenção de doenças, com vista à melhoria da qualidade de vida. ${ }^{24}$

Portanto, a falta de adesão às medidas de atenção à saúde é multifatorial e, para sua superação, é importante considerar a necessidade de uma escuta ampliada, a importância do estabelecimento de vínculo e o respeito pelo outro. Para isso, os profissionais da equipe precisam desenvolver a habilidade de cuidar da "pessoa" enquanto sujeito, com necessidades de saúde inerentes ao seu modo de vida, independentemente da doença que possa ser portador.

2. Assistência centrada no aspecto curativo e no atendimento à demanda

Para alguns entrevistados, a atenção à saúde ainda está voltada para os aspectos cura- tivos, centrada na consulta médica e no atendimento à demanda, mesmo frente à proposta da ESF em que atuam, a qual visa a uma abordagem ampliada das condições de vida e saúde das pessoas, família e comunidade, conforme se observa nas falas seguintes:

$$
\begin{aligned}
& \text { "É ainda muito curativa e centrada na figu- } \\
& \text { ra do médico. Quando uma unidade não tem } \\
& \text { um médico com o perfil de PSF fica ainda } \\
& \text { mais difícil a adesão”.(C3) } \\
& \text { “...no dia-a-dia a gente vê que a agenda é } \\
& \text { sempre lotada, muitos pacientes para atender, } \\
& \text { então muitas vezes a gente não consegue parar } \\
& \text { para ver o que está acontecendo...". (A2). }
\end{aligned}
$$

A dificuldade da ESF em contribuir para a ruptura do atual modelo pode estar relacionada à verticalização da proposta, à sua gestão centralizada e à homogeneidade na oferta do serviço, sem que sejam consideradas as diferenças regionais e o perfil epidemiológico, além da escassez de recursos humanos capacitados ou com perfil adequado. ${ }^{25}$

Franco e Merhry enfatizam que o problema do atual modelo de atenção à saúde está no processo de trabalho, restringindo-se à produção de procedimentos e não à produção do cuidado da cura. ${ }^{26}$

Observa-se que profissionais que atuam naESF, apesar de compor uma equipe, atuam de forma isolada, com agendas lotadas, sem que seja possível incluir a participação dos usuários, compartilhar saberes, trocar informações e definir estratégias conjuntas de enfrentamento dos problemas individuais e coletivos que possibilitem um atendimento 
voltado para as reais necessidades de saúde da população.

Quanto às ações que são desenvolvidas nas unidades, um dos entrevistados relata que

"... a ênfase está nas ações individuais, na consulta médica, de enfermagem, verificação da pressão arterial e entrega de medicamentos." (A3)

Tal verbalização indica ainda que a ESF tem como foco principal o atendimento à demanda, o que limita a possibilidade $d a$ "escuta qualificada", "do vínculo" e "da responsabilização", condições consideradas essenciais para haver a prática eficaz. Além disso, parece haver uma reprodução do modelo biologicista, desconsiderando a necessidade de implementação do modelo de vigilância da saúde, com ênfase na promoção da saúde.

Constituir tecnologias apropriadas para operar nos serviços de saúde é um grande desafio, pois exige um processo constante de criatividade frente à realidade local, de forma a combinar diferentes atos sanitários e atos clínicos ampliados, construídos cotidianamente pelo conjunto de profissionais da equipe. ${ }^{27}$

Capozzolo, ao referir-se ao trabalho do médico na atenção básica, discute sua complexidade tecnológica e as inúmeras tensões, ao considerar a necessidade de lidar ao mesmo tempo com normas e padronizações e com a singularidade do caso, combinar atividades programáticas e projetos terapêuticos individualizados, intervenções voltadas para diminuir riscos e, ao mesmo tempo, para aumentar os coeficientes de autonomia dos pacientes. ${ }^{28}$
Tais dificuldades aplicam-se também a toda equipe, acrescidas, em nossa vivência, de inúmeras atividades burocráticas, impostas principalmente ao agente comunitário de saúde como a necessidade de atender às regras e às normatizações.

Portanto, a tarefa de implantar a ESF demanda desenvolvimento contínuo das habilidades da equipe, pautado na interação entre os membros, destes com a comunidade e outros setores da sociedade, sustentando a constante reflexão sobre o processo de trabalho, já que cada realidade se apresenta como única, não cabendo receitas prontas.

A necessidade de avanços nos processos de atenção da ESF revela-se também nas narrativas seguintes:

\section{"As ações de saúde realizadas a qui na nossa unidade são relacionadas aos programas de saúde como o programa de hipertensão arterial e diabetes, prevenção de cancer bucal e cober- tura vacinal."(A3) \\ "...aqui na unidade é de qualidade, é como protocolo da Secretaria de Saúde, da coorde- nação e nós temos como prioridade o atendi- mento aos diabéticos, bipertensos, além dos idosos."(A1)}

Frente a tais narrativas, pode-se questionar como os dados do território e da população adscrita vêm sendo utilizados no planejamento das ações. É possível que tal condição esteja relacionada com o momento de transição, no qual as tecnologias necessárias para um processo de trabalho adequado ainda não foram devidamente apropriadas pelos 
profissionais da equipe. A própria formação de profissionais, apesar dos avanços, ainda não tem conseguido despertar o interesse deles, haja vista o esvaziamento das residências de medicina da família e comunidade.

$\mathrm{Na}$ percepção de alguns dos entrevistados, a assistência prestada na USF é de qualidade, por seguirem o protocolo de atendimento proposto pela Secretaria $\mathrm{Mu}$ nicipal de Saúde. Salienta-se, no entanto, que os protocolos existentes são destinados ao atendimento de portadores de hipertensão e diabetes e, que, apesar de a maioria ser idosa, também servem a outras faixas etárias. Além disso, tais protocolos, ao enfocar o cuidado de uma doença, demonstram-se insuficientes no atendimento integral da pessoa idosa.

Os idosos, por apresentarem características específicas, demandam, no atendimento das necessidades de saúde, busca ativa de seus riscos e danos, compreendendo os aspectos funcionais, sociais, emocionais e ambientais Tal busca deve fornecer elementos para a elaboração de propostas e desenvolvimento de ações visando a promoção, prevenção, cura e reabilitação das condições de saúde. Neste contexto, ênfase deve ser dada ao acolhimento, no acesso e à atenção global e interdisciplinar.

A complexidade da situação exige, então, a articulação da equipe, o desejo de enfrentamento dos problemas de forma interdisciplinar e capacitação dos profissionais para atuar frente às necessidades atuais, o que nos parece o grande desafio um ser enfrentado.

\section{Vislumbre de mudança no modelo} de atenção.

Nos depoimentos, ao considerar o que deveria ser realizado na ESF em prol da melhoria das condições de vida dos idosos, observa-se que os profissionais compreendem que ao idoso devem ser oferecidas oportunidades de participação, interação, educação, lazer e acesso às tecnologias de melhoria da qualidade de vida. Há também indicação da necessidade de alianças intersetoriais com o apoio dos profissionais da equipe de saúde, conforme descrito a seguir:

“... a gente pensa em fazer grupo de terapia ocupacional aqui na unidade, visando aos idosos, com brincadeiras, artesanato, dinâmica de grupo...". (G2)

"...o que falta para a gente acompanhar eles, é ter mais atividades com os idosos com parcerias com outras instituiçôes, proporcionar fisioterapia, educação física, ginástica, alongamento e atividades de lazer." (D1)

“...poderíamos desenvolver atividades educativas em relação às dietas e medidas não-medicamentosas."(D2)

"Mas também seria interessante... uma abordagem meio que de integração entre os idosos da área, como que servisse para eles passarem o tempo, aprenderem trabalhos manuais e até ganharem um dinheiro com isso que eles aprenderem, quem sabe?"(F1)

“... o acesso à prótese dentária deveria ser mais fácil. Isso melhoraria muito a qualidade de vida de alguns pacientes. Mas, além disso, 
precisamos de uma ação de conscientização dos mais jovens para que não cheguem a necessitar de uma prótese" (F3).

Ao considerar que a ESF pressupõe a substituição de práticas convencionais por um novo processo de trabalho centrado na vigilância à saúde e no trabalho interdisciplinar, "visando à melhoria progressiva das condiçoes de saúde e da qualidade de vida da população assistida", ${ }^{6}$ deslocando-se da atenção à doença para a atenção à saúde, pode-se compreender que os profissionais apresentam intencionalidades que vislumbram mudanças no modelo de atenção, cuja ênfase está na promoção da saúde.

A promoção da saúde vem sendo considerada, a partir da classificação desse conceito, em dois grandes grupos. Para o primeiro, "promocão da saúde compreende ampliar as oportunidades de escolha, alertar sobre as suas possiveis conseqüências, desenvolver trabalho educativo que incorpore a reflexão sobre a realidade vivida, estimulando a discussão sobre quais seriam as mudanças possiveis e instrumentalizando os profissionais com habilidades necessárias para a realização das mudanças desejadas". Neste ponto de vista, a promoção da saúde consiste em atividades dirigidas à transformação dos comportamentos dos indivíduos, focando seu estilo de vida e atividades educativas, visando a atuar frente a condições passíveis de serem modificadas pela própria vontade da pessoa. ${ }^{6} \mathrm{O}$ segundo grupo refere-se a um conceito mais ampliado, ao considerar que "promoção da Saúde parte de uma concepção ampla do processo saúde-doença e de seus determinantes epropõe a articulação de saberes técnicos e populares e a mobilização de recursos institucionais e comunitários, públicos e privados, para seu enfrentamento e resolu- ção. ${ }^{6}$ Trata-se de um conceito que diversifica as necessidades de intervenção e demanda políticas intersetoriais. ${ }^{6}$ As ações que as equipes vêm desenvolvendo parecem ter, portanto, maior relação com o primeiro grupo.

Merece ser destacada, nas proposições dos profissionais que atuam na ESF, a oportunidade de ampliar ações para fortalecer os potenciais de cuidados com a saúde. No âmbito da educação em saúde, a discussão da autonomia sempre tem estado presente como perspectiva de processos emancipatórios voltados para o fortalecimento dos sujeitos. ${ }^{29}$ Zaboli defende que as obrigações dos profissionais de saúde incluem atuar para ampliar a capacidade de escolha superando as barreiras de informações que perpetuam medos e cristalizam tabus. ${ }^{30}$

Acrescenta-se, ainda, a ênfase dada aos processos grupais. A atenção realizada em grupos facilita o exercício da autodeterminação e da independência, pois o grupo pode funcionar como rede de apoio que mobiliza as pessoas na busca de autonomia e sentido para a vida, na auto-estima e, até mesmo, na melhora do senso de humor, aspectos essenciais para essa ampliação. ${ }^{31}$

$\mathrm{Na}$ fala dos sujeitos que apontam para as ações que visam a uma atenção adequada à saúde do idoso, pode-se constatar, ainda, que a atual conformação da equipe básica da ESF não é suficiente para o atendimento dessa parcela da população, havendo evidências da necessidade de comporem a equipe profissionais como o educador físico, nutricionista, fisioterapeuta e terapeuta ocupacional. 
Além disso, na perspectiva de um atendimento integral à saúde do idoso, visando principalmente à promoção da saúde, a concretização das mudanças vai além das possibilidades dos profissionais da saúde. É preciso despertar outros setores da sociedade, como os da educação, lazer, esporte e transporte, o que demanda vontade política, conscientização dos atores e definição de estratégias capazes de atender à complexidade que representa melhorar as condições de vida dos idosos.

\section{CONSIDERAÇÕES FINAIS}

A atenção à saúde do idoso reveste-se de grande complexidade, principalmente quando a assistência é direcionada por um conceito ampliado do processo saúde/doença, com vistas à melhoria da qualidade de vida. Atuar desta forma é o desafio que se coloca aos serviços de saúde, em especial, às equipes $d a$ ESF que vêm sendo implantadas em todo o território nacional.

Ao analisar as falas dos profissionais que atuam naESF, referentes à sua percepção quanto à saúde do idoso, bem como às ações que desenvolvem e o que consideram que poderiam realizar em prol da melhoria das condições de saúde dos idosos, foi possível identificar três categorias de análise: "Falta de adesão ao cuidado e de apoio dos familiares”; "Assistência centrada no aspecto curativo e no atendimento a demanda" $e$ "V islumbre de mudança no modelo de atenção".

Tais categorias evidenciam o momento de transição que se encontra o sistema de saúde brasileiro, em que há ações que permeiam o velho modelo e já se percebem algumas mudanças e desejos de percorrer um caminho que vislumbre a melhoria da qualidade de vida das pessoas, mesmo com as dificuldades impostas pela restrita concepção de saúde-doença que ainda predomina entre a população e profissionais de saúde.

Esta condição contribui para a reflexão da ESF como o caminho de consolidação do SUS, pois se acredita que, a partir dos desejos, as ações vão se delineando e, assim, a ESF pode constituir um instrumento de mudanças.

Caminhar na direcionalidade da construção de um novo modelo significa compreender a complexidade que a denominada "rede básica” ou "atenção primária” está trabalhando atualmente, tendo em vista o envelhecimento da população.

Constata-se, então, que, para atuar junto a essa parcela da população é necessário pautar-se na integralidade do cuidado e na lógica da vigilância da saúde visando a promoção, prevenção, cura e reabilitação das condições de saúde. Neste contexto, o papel dos profissionais de saúde assume um dimensionamento ampliado e, muitas vezes, distinto das bases de formação que por muitos anos vem privilegiando o tecnicismo e deixando margens pouco definidas e, portanto, conflituosas, ao implementar o trabalho interdisciplinar.

Nesta trajetória, no entanto, é preciso considerar a necessidade de contínuos investimentos na capacitação dos profissionais, visando à abordagem multidimensional e 
interdisciplinar da pessoa idosa e tendo como eixos norteadores para a integralidade de ações: o enfrentamento de fragilidades da pessoa idosa, da família e do sistema de saúde e a promoção da saúde e da integração social, em todos os níveis de atenção, conforme proposto no pacto pela saúde do idoso. ${ }^{12}$

\section{NOTAS}

\author{
Doutora em Enfermagem, aluna do Programa de Pós- \\ Graduação em Ciências da Saúde, nível pós-doutorado, \\ da Universidade Federal de São Paulo. Docente da dis- \\ ciplina de Enfermagem em Saúde Coletiva da Faculda- \\ de de Medicina de Marília. \\ b Estudante do Curso de Medicina da Faculdade de Me- \\ dicina de Marília. Bolsista de Iniciação Científica pela \\ FAPESP. \\ c Estudante do Curso de Enfermagem da Faculdade de \\ Medicina de Marília. \\ d Especialista em Saúde da Família e enfermeira do Pro- \\ grama de Saúde da Família do Município de Marília. \\ Enfermeira, bolsista de Treinamento Técnico da \\ FAPESP.
}

\section{REFERÊNCIAS}

1. Chaimowicz F. A saúde dos idosos brasileiros às vésperas do século XXI: problemas, projeções e alternativas. Rev Saúde Pública 1997; 31(2): 184-200.

2. Garrido R, Menezes PR. O Brasil está envelhecendo: boas e más notícias por uma perspectiva epidemiológica. Rev Bras Psiquiatr 2002: 24(supl.1):3-6.

3. Silva Junior JB, Gomes FBC, Cezário AC, Moura L. Doenças e agravos nãotransmissíveis: bases epidemiológicas. In: Rouquayrol MZ, Almeida Filho N. Epidemiologia \& saúde. 6. ed. Rio de Janeiro: Medsi; 2003. p.289-311.

4. World Health Organization. Envelhecimento ativo: uma política de saúde. Brasília: Organização Pan-Americana da Saúde; 2005.

5. Buss. Promoção e educação em saúde no âmbito da Escola de Governo em Saúde da Escola Nacional de Saúde Pública. Cad Saúde Pública 1999; 15(supl. 2):177-85.

6. Buss PM. Promoção da saúde e qualidade de vida. Ciência Saúde Coletiva 2000; 5(1):163-77.
7. Noronha AB. Graduação: é preciso mudar. Radis: comunicac'aPo em sauide 2002; (5): 9-16.

8. Brasil. Ministério da Saúde. Fundação Nacional de Saúde. Programa de Saúde da Família: saúde dentro de casa. Brasília; 1994.

9. Brasil. Ministério da Saúde. Secretaria de Assistência à saúde. Coordenação de saúde da comunidade. Saúde da Família: uma estratégia para reorientação do modelo assistencial. Brasília; 1998.

10. Brasil. Ministério da Saúde. Política Nacional de Humanização: Humaniza SUS -

Documento base para gestores e trabalhadores do SUS. Brasília, 2006. 52 p. (Serie $\mathrm{B}$ textos básicos de saúde). Disponível em: URL: http://portal.saude.gov.br/ portal/arquivos/pdf/DB_PNH.pdf

11. Brasil. Portaria n. 399/GM, de 22 de fevereiro de 2006. Divulga o Pacto pela Saúde 2006 Consolidação do SUS e aprova as Diretrizes Operacionais do Referido Pacto. Disponível em: URL: http://portal.saude.sp.gov.br/ resources/gestor/destaques/ pacto_portaria_399_06.pdf 
12. Brasil. Portaria n. 2.528 de 19 de outubro de 2006. Aprova a Política Nacional de Saúde da Pessoa Idosa. Disponível em: URL: http://portal.saude.gov.br/portal/arquivos/ pdf $/ 2528 \%$ 20aprova\% 20a\% 20politica\% 20nacional $\% 20$ de $\% 20$ saude $\% 20 \mathrm{da} \%$ 20pessoa\%20idosa.pdf

13. Silvestre JA, Costa Neto MM. Abordagem do idoso em programas de saúde da família. Cad Saúde Pública 2003; 19(3): 839-47.

14. Araujo MAS, Nakatani AYK, Silva LBS, Bachion MM. Perfil do idoso atendido por um programa de saúde da família em Aparecida de Goiânia. Revista da UFG 2003 dez; 5(2). [citado em 2006 set 28]; Disponível em:URL: http://www.proec.ufg.br/ revista_ufg/idoso/perfil.html

15. Bezerra AFB, Espírito Santo, ACG, Batista Filho M. Concepções e práticas do agente comunitário na atenção à saúde do idoso. Rev Saúde Pública 2005; 39(5): 809-15.

16. Lopes FAM, Oliveira FA. Aspectos epidemiológicos de idosos assistidos pelo Programa de Saúde da Família (PSF) [online]. Uberaba: Universidade Federal do Triângulo Mineiro; 2004. [acesso em 2006 jun 26]. Disponível em: URL: http://www.fmtm.br/ instpub/fmtm/patge/Idoso_psf.htm

17. Cunha MI. Conta-me agora!: as narrativas como alternativas pedagógicas na pesquisa e no ensino. Revista da Faculdade de Educação da Universidade de São Paulo 1997; 23(1-2):185-95.

18. Bardin L. Análise de conteúdo. São Paulo; 1977

19. Minayo MCS. O desafio do conhecimento: pesquisa qualitativa em saúde. 6. ed. São Paulo: Hucitec; Rio de Janeiro: Abrasco; 1999.269 p.

20. Silveira LMC, Ribeiro VMB. Grupo de adesão ao tratamento: espaço de "ensinagem" para profissionais de saúde e pacientes. Interface 2004/2005; 16(9): 91-104.
21. Fuertes JN, Mislowack A, Bennett J, Paul L, Gilbert TC, Fontan G, Boylan LS. The physician-patient working alliance. Patient Educ Couns 2007: 66 (1): 29-36.

22. Osterberg L, Blaschke T. Adherence to medication. N Engl J Med 2005; 353(5):487-97.

23. Damatta R. A casa e a rua: espaço, cidadania, mulher e morte no Brasil. 4. ed. Rio de Janeiro: Guanabara Koogan; $1991.177 \mathrm{p}$.

24. Lopes ESL, Néri AL, Park MB. Ser avós ou ser pais: Os papéis dos avós na sociedade contemporânea. Textos Envelhecimento 2005: 8(2) Disponível em: URL: http://www.unati.uerj.br/tse/ scielo.php?script $=$ sci_arttext\&pid $=$ S1517$592820050002000068 \overline{l n} \mathrm{ng}=$ pt\&nrm = iso.

25. Scherer MDA, Marino SRA, Ramos F. Rupturas e resoluções no modelo de atenção à saúde: reflexões sobre a estratégia de saúde da família com base nas categorias kuhnianas. Interface 2004-2005; 53-66.

26. Franco T, Merhy EE. PSF: contradições e novos desafios [online]. 1999. [citado em 2007 dez 21]. Disponível em: URL: http:// www.datasus.gov.br/cns

27. Campos GVS. Análise crítica das contribuições da saúde coletiva à organização das práticas de saúde no SUS. In: Fleury S, organizador. Saúde e democracia: a luta do Cebes. São Paulo: Lemos Editorial; 1997. p. 113-25.

28. Capozzolo AA. No olho do furação: trabalho médico e o Programa de Saúde da Família [tese]. Campinas: Universidade de Campinas; 2003.

29. Chiesa AM. Autonomia e resiliência: categorias para o fortalecimento da intervenção na atenção básica na perspectiva da promoção da saúde [tese]. São Paulo: Universidade de São Paulo; 2005. 
30. Zaboli ELCP. Bioética e atenção básica: um estudo de ética descritiva com enfermeiros do Programa de Saúde da Família [tese]. São Paulo: Universidade de São Paulo; 2003.
31. Moreira MMS. Trabalho, qualidade de vida e envelhecimento [dissertação]. Rio de Janeiro: Escola Nacional de Saúde Pública; 2000.

Recebido em: 25/2/2008

Reapresentado: 26/3/2008

Aprovado: 30/4/2008 